\title{
BIBECHANA
}

ISSN 2091-0762 (Print), 2382-5340 (Online)

Journal homepage: http://nepjol.info/index.php/BIBECHANA

Publisher: Department of Physics, Mahendra Morang A.M. Campus, TU, Biratnagar, Nepal

\section{A spectroscopic study of the low redshift dwarf galaxy SDSS J134326.99+431118.7 to calculate star formation rate}

\author{
Daya Nidhi Chhatkuli ${ }^{1 *}$, Sanjaya Paudel $^{2}$, Binil Aryal ${ }^{1 * *}$ \\ ${ }^{1}$ Central Department of Physics, Tribhuvan University, Nepal. \\ ${ }^{2}$ Centre for Galaxy Evolution Research, Department of Astronomy, Yonsei University, Seoul, South \\ Korea. \\ "Email: chhatkulidn@gmail.com \\ ***Eail: binil.aryal@cdp.tu.edu.np
}

\section{Article Information:}

Received: June 15, 2020

Accepted: July 10, 2020

Keywords:

Dwarf galaxy

Star formation rate

Characteristic peaks

$H_{\alpha}$ line

\begin{abstract}
We present a spectroscopic study of an interacting emission-line dwarf galaxy SDSS J134326.99+431118.7. We analyzed eight-strong emission lines of wavelength in a range of $3902.1 \AA$ to $6619.1 \AA$. Among them, the strongest emission line is OIII, with an intensity of $1043.6 \times 10^{-17} \mathrm{erg} / \mathrm{s} / \mathrm{cm}^{2} / \AA$. These characteristic lines show a perfect Gaussian fit with a coefficient of regression greater than $98 \%$, where the derived full width half maximum (FWHM) is less than $3.8 \AA$. The line ratio between $\mathrm{H}_{\alpha}$ and $\mathrm{H}_{\beta},\left(\mathrm{H}_{\alpha} / \mathrm{H}_{\beta}\right)$, is 2.73. This suggests that the galaxy is a starburst galaxy. Star Formation Rate (SFR) of the galaxy derived from $\mathrm{H}_{\alpha}$ emission line flux is $0.019 \mathrm{M}_{\odot}$ year $^{-1}$ and emission line metallicity derived from flux ratio between NII and $\mathrm{H}_{\alpha}$ is 7.85 dex. These morphological and physical properties of SDSSJ134326.99+431118.7 are very similar to those of a typical Blue Compact Dwarf (BCD) galaxy. We conclude that we have presented another evidence of forming a BCD-type galaxy through a merger.
\end{abstract}

DOI: https://doi.org/10.3126/bibechana.v18i1.29466

This work is licensed under the Creative Commons CC BY-NC License. https://creativecommons.org/licenses/by-nc/4.0/

\section{Introduction}

A galaxy whose gravitational fields interact with another and produce a disturbance in their morphological and dynamical properties are called interacting galaxies [1]. There are many observational pieces of evidence that link galaxies' morphological and physical evolution with interactions and mergers. Starburst activities are frequent among the interacting galaxies, particularly in low mass cases where a vast amount of gas reservoir is present. The gravitational tidal force of the interacting system destroys the regular 
morphology of the participating galaxies and produces bridges and tails between them [2]. This also destabilizes gaseous disk leading to an inflow of star-forming gas toward the central region, which creates a nuclear starburst $[3,4]$.

According to Lambda Cold Dark Matter $(\Lambda \mathrm{CDM})$ cosmology, dwarf galaxies are the building blocks of the universe, and all large-scale structures are build up progressively later with the hierarchical coalescence of these building blocks. They dominate the number distribution at any epoch of the universe, and there should be a more frequent merger between dwarf galaxies than between massive galaxies. However, the dwarf-dwarf interactions are not explored in detail, as in the case of massive galaxies' interactions. On the other hand, dwarf galaxies are inherently low-surface brightness in nature, and it is challenging to detect them. Because of this, it is not trivial to detect tidal features in dwarf galaxies, which are even fainter than host galaxies.

There is a great deal of the study of the interacting massive galaxies, but it is less known about the interaction and mergers of the dwarf galaxies to date [5]. It is also true that the merging probability of dwarf galaxies is low at low redshift universe as most of them are bound within the gravity of large scale structures, where dispersion velocities are high [6]. Because of this, dwarf-dwarf interactions are more frequent in the field environment than in the group environment where galaxies are more gas-rich compare to dwarf galaxies located in the group environment [7]. Furthermore, dwarf galaxies merge frequently and assemble due to collision to make bigger galaxies. The growth and evolution of the galaxies is based on the merger of dwarf galaxies [8]. Therefore, it is interesting to study the dwarf-dwarf merger at a low redshift regime and understand the effect of the merger in gas removal and star-formation in these low-mass systems. It is not conclusively understood how important a merger is in dwarf galaxy evolution.

Stierwalt et al. (2015) [9] is the first to perform a statistical study of interacting dwarf galaxy pairs and found that star formation is more intensified in paired dwarfs than that in unpaired galaxies. They found that star-formation rates of interacting dwarf galaxies are anti-correlated with their pair separation. Moreover, a significant fraction of starburst in dwarf galaxies are contributed by the interaction. Lelli et al. (2018) suggest that the activity of the starburst galaxies such as blue compact dwarfs (BCDs) strongly depends on the inner shape of the potential well and the central gas surface density [10].

In this work, we present the spectroscopic study of a star-forming interacting dwarf galaxy SDSS J134326.99+431118.7, a morphologically blue and edge-on dwarf galaxy. This galaxy is located in a nearly isolated environment where we find no massive galaxies within a $500 \mathrm{kpc}$ radius around it.

\section{Sample selection}

SDSS J134326.99+431118.7 is listed in the catalog of interacting dwarf galaxies by Paudel et al. (2018) [11]. It is located in the sky position $R A=13 \mathrm{~h} 43 \mathrm{~m}$ $26.9 \mathrm{~s}$ and $\mathrm{Dec}=43 \mathrm{~d} 11 \mathrm{~m} 18.78 \mathrm{~s}$ with a line-ofsight radial velocity $2511(\mathrm{Z}=0.00837) \mathrm{km} / \mathrm{s}$. It has $\mathrm{g}$ and $\mathrm{r}$ band magnitude $16.18 \mathrm{mag}$ and 15.98 mag respectively, and total absolute B-band magnitude is -15.34 mag. In Figure 1, we show the $g-r-i$ combined tri-color image obtained from the Legacy survey. We can see a shell feature morphology at the lower side with an extended arclike feature at the sky projected distance of $5 \mathrm{kpc}$ from the center. The central part is significantly blue compare to the outer extended low-surface brightness region. A red cross mark identifies an off-centered star-burst region, where the SDSS has positioned its fiber to take the optical spectrum.

In Figure 2, we show the SDSS optical spectrum of the wavelength range of 4000 to $6600 \mathrm{~A}$. We can see several characteristic lines in the spectrum and highlighted some of the prominent emission lines, mainly Balmer lines. The strongest emission line is OIII, and $\mathrm{H}_{\alpha}$ is the strongest among the Balmer lines. 


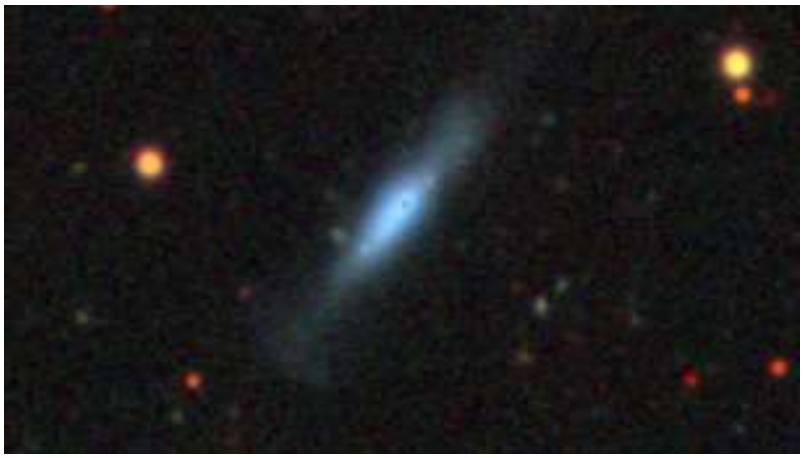

Fig. 1: Optical view of SDSS J134326.99+431118.7. The image is obtained from the SDSS sky-server.

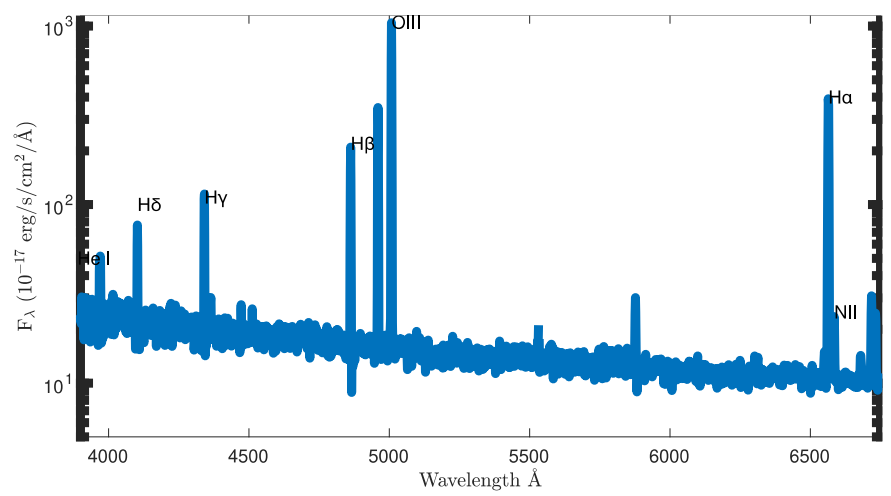

Fig. 2: Optical spectrum of SDSS J134326.99+431118.7. Positon of the emission lines $\mathrm{H}_{\alpha}, \mathrm{H}_{\beta}, \mathrm{H}_{\gamma}, \mathrm{H}_{\delta}, \mathrm{OIII}, \mathrm{HeI}$ and NII are identified. The $\mathrm{X}$-axis is rest-frame wavelength and $\mathrm{Y}$-axis is flux.

\section{Data analysis}

In this work, we extensively use the SDSS archival data to measure morphological and chemical parameters of the galaxy. We obtained the SDSS g and $r$ band images and optical fiber-spectrum from the SDSS Data Archive Server (DAS). The images and spectrum are already well-calibrated, and we further do not perform any operation in this regard. Both images and spectrum data are in the fits file format. The SDSS fiber has a diameter of 3 arc seconds. Hence, the observed spectrum only covers a small portion of the galaxy area. The SDSS optical spectrum has coverage of 3650 to $10,400 \AA$, which includes primary emission and absorption lines that are frequently used to investigate the chemical properties of stars or galaxies. The spectral resolution of the SDSS spectrum is 1500 at $3800 \AA$.

The spectrum of the galaxy was analyzed by using the Origin software of the 18th version. We first identified 21 different emission lines in the spectrum as shown in Figure 2. We performed Gaussian fits in each emission line and measured their parameters such as Full Width Half Maximum (FWHM), total flux, and best-fit coefficient. Before modeling each emission line, we subtracted the continuum flux taking a median of flux value of spectrum range \pm 100 from the center of the emission line.

Emission line profile of the extragalactic object is dominated by Doppler broadening. The atoms/molecules in a gas, which emits the radiation, will have a distribution of velocities. The photon emitted by transition will be either red or blue-shifted by the Doppler effect, depending on the atom's velocity relative to the observer. Since the spectral line is a combination of all of the emitted radiation, the line profile is considered a Gaussian in nature. The center is zero shift or an average shift of the system. Mathematically Gaussian distribution function is defined as [12]

$$
\mathrm{f}_{\mathrm{G}}(\mathrm{x})=\frac{1}{\sqrt{2 \pi \sigma^{2}}} \mathrm{e}^{\frac{-(\mathrm{x}-\mu)^{2}}{2 \sigma^{2}}}
$$

Where, $x$ is a normal random variable, $\mu$ is the mean deviation and $\sigma$ is the standard deviation of the distribution. The FWHM is a width at which maximum amplitude drops to the half. We selected eight emission lines based on a higher intensity, which will be presented in this paper, along with their Gaussian parameters. The Gaussian parameters will be enlisted in a separate table and the corresponding elements will be identified. Moreover, we calculate the star formation rate (SFR) and the hydrogen line ratio. We will use the Gaussian area of $\mathrm{H} \alpha$ emission line to calculate the star formation rate of the galaxy. We calculate star 
formation rate adopting the empirical formula proposed by Kennicutt (1998) [13] as follows:

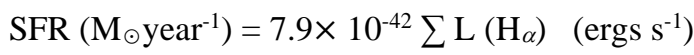

where $\sum \mathrm{L}(\mathrm{H} \alpha)$ is the total luminosity of $\mathrm{H} \alpha$ line which will be calculated by using Gaussian fits. $\sum \mathrm{L}(\mathrm{H} \alpha)=$ Area of Gaussian fit $\times 10^{-17} \times$ $4 \pi \mathrm{R}^{2}\left(\operatorname{ergs~s}^{-1}\right) . \mathrm{R}$ is the radius of the sphere which is calculated as $\mathrm{R}=\mathrm{D} \times 3.08 \times 10^{24} \mathrm{~cm}$. Here, $\mathrm{D}$ is luminosity distance of the galaxy in Mpc.

We derive emission line metallicity using a line ratio between $\mathrm{H}_{\alpha}$ and NII. We use a calibration provided by Marino et al.(2013) [14].

$12+\log (\mathrm{O} / \mathrm{H})=8.743+0.462 \times \log \left(\mathrm{NII} / \mathrm{H}_{\alpha}\right)$

\section{Results}

In this work we have analyzed eight main emission lines, i.e., $\mathrm{HeI}, \mathrm{H}_{\delta}, \mathrm{H}_{\gamma}, \mathrm{H}_{\beta}$, OIII, OIII, $\mathrm{H}_{\alpha}$, and NII which are identified in Figure 2. The OIII is a doublet. Therefore, it has two different emission lines. The $\mathrm{H}_{\delta}, \mathrm{H}_{\gamma}, \mathrm{H}_{\beta}$, and $\mathrm{H}_{\alpha}$ are Balmer lines and they are sensitive to the galaxies' gas-mass and star-formation rate. The $\mathrm{H}_{\alpha}$, principally, is a well-known tracer of star-formation and widely used to calculate the star-formation rate of the gas-rich galaxies. Heavier elements OIII and NII emission fluxes are used to calculate the star-forming galaxies' emission line metallicity.

In Figure 3, we show a few examples of the Gaussian fitting procedure. Here, we selected four main examples of emission lines of the spectra of interacting dwarf galaxy SDSS J134326.99+431118.7. We list peaks' position and the central wavelength value of all eight emission lines in Table 1. The solid line represents the Gaussian distribution. The error bars are also shown in the Gaussian curves. We can see that there is the almost perfect agreement of the observed data with the Gaussian distribution.
We list all eight strongest emission lines in the spectrum of the galaxy SDSS $\mathrm{J} 134326.99+431118.7$ that we have studied in this work in table 1 . Their maximum intensity, the wavelength corresponding to maximum intensity, and corresponding elements are also provided. From the table, we can see that the doubly ionized oxygen OIII has a maximum intensity of $1043.6 \mathrm{x}$ $10^{-17} \mathrm{erg} / \mathrm{s} / \mathrm{cm}^{2} / \AA$, which has a central wavelength $5050.1 \AA$. The second strongest intensity $(391.8 \mathrm{x}$ $10^{-17} \mathrm{erg} / \mathrm{s} / \mathrm{cm}^{2} / \AA$ ) is found corresponding to the wavelength $6619.1 \AA$, which is $H_{\alpha}$ line. The weakest intensity $\left(31.34 \times 10^{-17} \mathrm{erg} / \mathrm{s} / \mathrm{cm}^{2} / \AA\right)$ among the eight emission lines corresponds to the wavelength $6634.23 \AA$ for the NII.

The Gaussian parameters of all eight Gaussian distributions are listed in table 2. The Gaussian height is given in the unit of intensity. Gaussian offset is the positional difference between the Gaussian peak and the observed peak in the spectra. The coefficient of regression represented by $\mathrm{R}$ square is found to be more than 0.980. This suggests a perfect Gaussian fit with the characteristic lines.

\section{Calculation}

Star Formation Rate can be calculated by using equation (2). Here, we take $\mathrm{D}=35.27 \mathrm{Mpc}$ from the catalogue prepared by Paudel et al. (2018) for this particular galaxy.

Now, $\mathrm{R}=\mathrm{D} \times 3.08 \times 10^{24} \mathrm{~cm}$

$$
\begin{aligned}
& =35.27 \times 3.08 \times 10^{24} \mathrm{~cm} \\
& =108.63 \times 10^{24} \mathrm{~cm}
\end{aligned}
$$

Star formation rate due to $\mathrm{H} \alpha$ line is

$\operatorname{SFR}\left(M_{\odot}\right.$ year $\left.^{-1}\right)=7.9 \times 10^{-42} \times \quad$ Area of

Gaussian fit $\times 10^{-17} \times 4 \pi \mathrm{R}$

$=7.9 \times 10^{-42} \times 1661.01 \times 10^{-17} \times 4 \pi \times(108.63 \times$

$\left.10^{24}\right)^{2}=0.019 \mathrm{M}_{\odot}$ year $^{-1}$

Line ratio $H \alpha / H_{\beta}=2.73$. This is slightly lower than the theoretical value of $\mathrm{H} \alpha / \mathrm{H}_{\beta}=2.8$. We suspect that this discrepancy between the theoretical and observed value is due to the inaccurate subtraction of continuum in the region of 

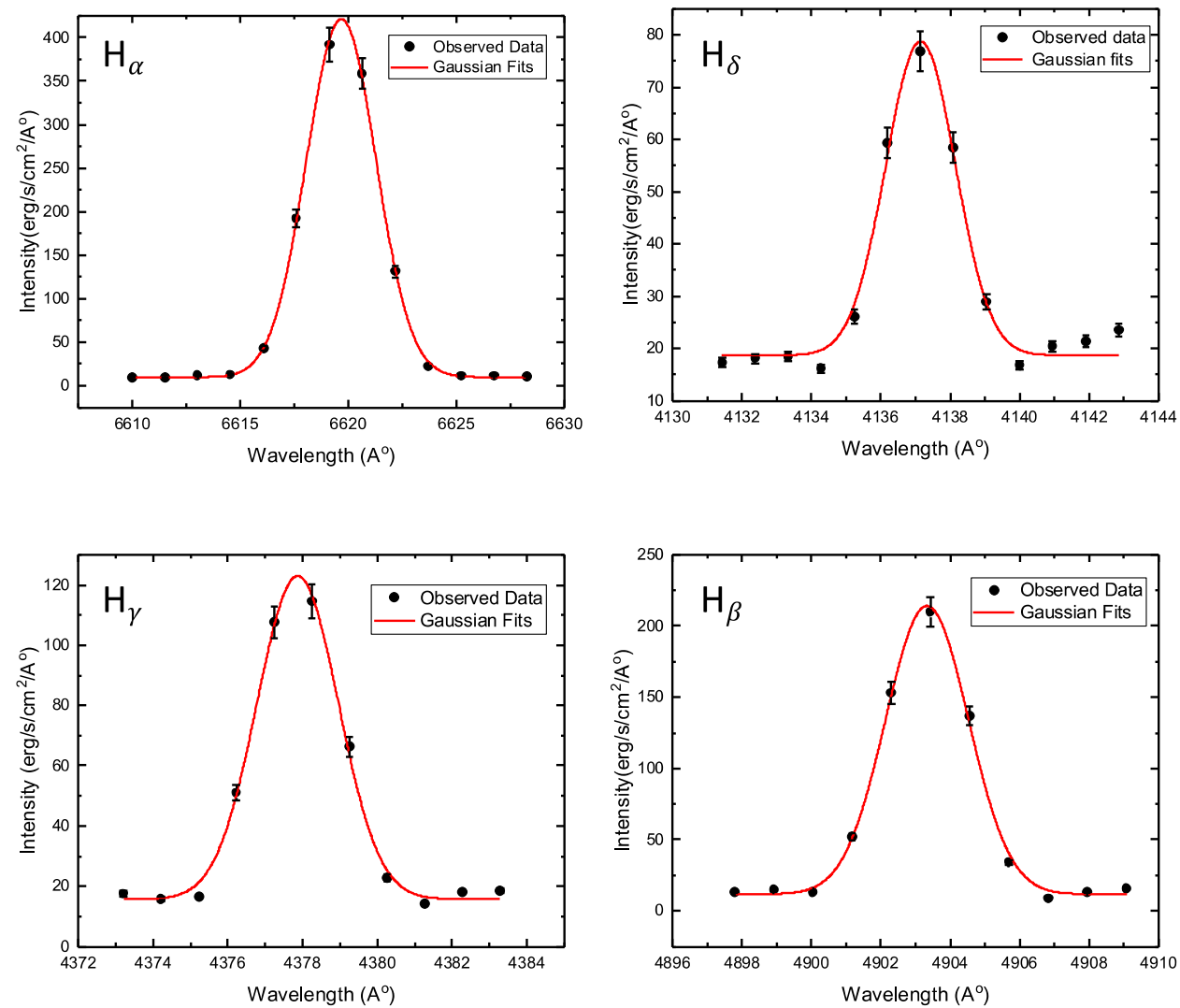

Fig. 3: We show four main examples of Gaussian fitting procedure. They are four Balmer lines: $\mathrm{H}_{\alpha}$. $\mathrm{H}_{\delta}$, $\mathrm{H}_{\psi}$, and $\mathrm{H}_{\beta}$. Solid curves represent Gaussian fits. We show a conservative estimate of the flux error in the plot, i.e., $10 \%$ of the observed flux provided by SDSS webpage (https://www.sdss.org/dr15/spectro/caveats/). The major cause of broadening of characteristic line is due to the Doppler broadening. The wavelengths given in the $\mathrm{X}$-axis are not redshift corrected.

Table 1: The selected characteristic emission lines of spectra of dwarf galaxy SDSS J134326.99+431118.7 shown in Figure 3 are described along with their physical parameters listed in the table. The first column represents the name of the selected emission lines. Second and third columns represent the wavelength $\left(\left(\lambda_{\mathrm{p}}\right)\right.$ corresponding to the peak intensity, the value of peak intensity (Ip).

\begin{tabular}{|c|c|c|}
\hline $\begin{array}{c}\text { Responsible } \\
\text { Elements for } \\
\text { Emission Lines }\end{array}$ & $\begin{array}{c}\text { Wavelength } \\
\text { Corresponding to } \\
\text { Peak Intensity } \\
\boldsymbol{\lambda}_{\mathbf{p}}(\mathbf{\AA})\end{array}$ & $\begin{array}{c}\text { Peak Intensity } \\
\mathbf{I}_{\mathbf{p}} \\
\left(\mathbf{1 0}^{-\mathbf{1 1 7}} \mathbf{e r g} / \mathbf{s e c} / \mathbf{c m}^{2} / \mathbf{\AA}\right)\end{array}$ \\
\hline $\mathrm{HeI}$ & 3921.9 & 64.8 \\
\hline $\mathrm{H}_{\delta}$ & 4137.1 & 76.8 \\
\hline $\mathrm{H}_{\gamma}$ & 4378.2 & 114.6 \\
\hline $\mathrm{H}_{\beta}$ & 4903.4 & 209.8 \\
\hline $\mathrm{OIII}$ & 5001.5 & 349.7 \\
\hline $\mathrm{OIII}$ & 5050.1 & 1043.6 \\
\hline $\mathrm{H}_{\alpha}$ & 6619.1 & 391.8 \\
\hline $\mathrm{NII}$ & 6634.23 & 31.34 \\
\hline
\end{tabular}


Table 2: Full width half maximum, area of the curve, height of the Gaussian curve, offset and coefficient of regression of the data corresponding to each selected spectral lines are enlisted in the table in second, third, fourth, fifth and sixth column respectively corresponding to the responsible elements.

\begin{tabular}{|c|c|c|c|c|c|}
\hline Element & $\begin{array}{c}\text { FWHM } \\
(\AA)\end{array}$ & $\begin{array}{c}\text { Area } \\
\left(10^{-17}\right. \\
\left.\mathrm{erg} / \mathrm{s} / \mathrm{cm}^{2} / \AA\right)\end{array}$ & $\begin{array}{c}\text { Height } \\
\left(10^{-17}\right. \\
\text { erg/s/cm²/A })\end{array}$ & Offset & R-square \\
\hline $\mathrm{HeI}$ & 2.23 & 117.49 & 49.39 & -0.253 & 0.980 \\
\hline $\mathrm{H}_{\delta}$ & 2.35 & 150.05 & 59.92 & -0.008 & 0.984 \\
\hline $\mathrm{H}_{\gamma}$ & 2.57 & 292.96 & 107.25 & +0.376 & 0.995 \\
\hline $\mathrm{H}_{\beta}$ & 2.82 & 606.93 & 202.38 & +0.099 & 0.997 \\
\hline OIII & 2.69 & 1003.65 & 350.56 & -0.252 & 0.998 \\
\hline OIII & 2.68 & 2982.67 & 1045.33 & -0.001 & 0.999 \\
\hline $\mathrm{H}_{\alpha}$ & 3.80 & 1661.01 & 411.18 & -0.558 & 0.999 \\
\hline NII & 3.80 & 19.30 & 32.40 & +0.023 & 0.999 \\
\hline
\end{tabular}

$\mathrm{H}_{\beta}$, where we can see absorption feature is also present. The ratio between $\mathrm{H} \alpha$ and $\mathrm{H}_{\beta}$ are also used to check the presence of internal dust reddening in the galaxies. The observed line ratio is near to the theoretical value or less. This imply that

SDSSJ134326.99+431118.7 is a nearly dust free galaxy.

Line ratio NII/ $\mathrm{H}_{\alpha}=0.011$. We calculate emission line metallicity for this galaxy using equation (3), $12+\log (\mathrm{O} / \mathrm{H})=7.85$ dex. Logarithmic value is represented by dex. It is especially suitable while talking about metallicity. Metallicity is measured logarithmically in connection with the plenty of metals in the galaxy.

\section{Discussion}

Table 2 shows that the selected Gaussian distributions of $\mathrm{HeI}, \mathrm{H}_{\delta}$, OIII doublet and $\mathrm{H} \alpha$ have a negative value of the offset. These curves have negative skewness. This means that the left-hand tail is longer than that on the right-hand side, and the mode is more than the mean. Similarly, the distributions of $\mathrm{H}_{\gamma}, \mathrm{H}_{\beta}$ and NII have a positive offset. The curves have positive skewness. This means that the right-hand tail is longer than that of the left-hand side, and the mode is less than the mean. The value of FWHM is maximum (3.80 $\AA$ ), corresponding to the curve of $\mathrm{H}_{\alpha}$. This indicates that the observed data is in excellent agreement with the Gaussian fit. The Gaussian area is maximum $\left(2982.67 \times 10^{-17}\right.$ $\mathrm{erg} / \mathrm{s} / \mathrm{cm}^{2} / \AA$ ) for the line OIII.

Normal galaxies follow scaling relations of B-band absolute magnitude $(\mathrm{Mb})$ versus star-formation rate and $\mathrm{Mb}$ versus emission-line metallicity. The position of SDSSJ134326.99+431118.7 in these relations is shown in Figure 4. In this figure, we have taken the SDSS star-forming galaxies as a comparative sample for the magnitude-metallicity relationship and the Local volume star-forming galaxies studied by Lee et al. (2009) [15] for the magnitude-star-formation rate relation. Interestingly, we find that SDSSJ134326.99+431118.7 is a significant outlier. It is significantly metal-poor compare to the normal SDSS star-forming galaxies. Similarly, the starformation activity in this galaxies is notably higher than that of normal star-forming galaxies of local volume.

The catalog value of the star-formation rate of SDSSJ134326.99+431118.7 is $0.0083 \mathrm{M}_{\odot}$ year $^{-1}$, which is derived by using the Far-Ultraviolet (FUV) flux of the galaxy. This shows that our 

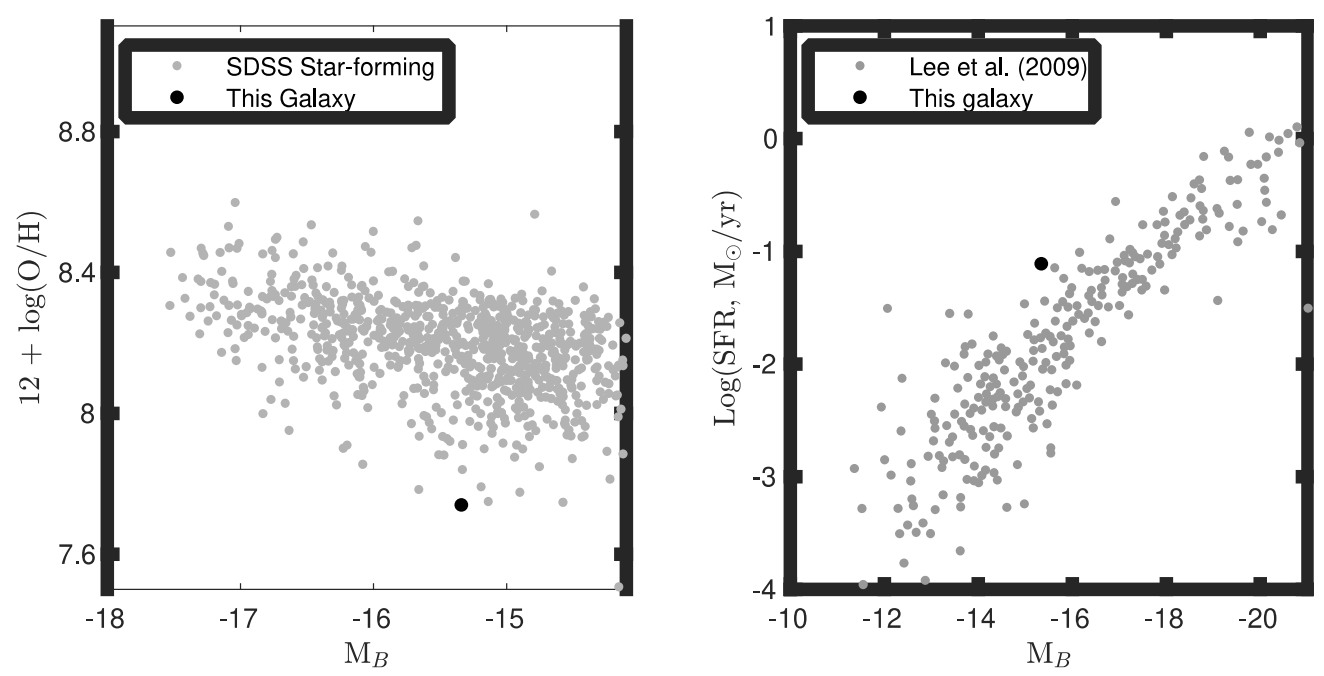

Fig. 4: Magnitude-metallicity relation of star-forming galaxies, left, where the SDSS star-forming galaxies as a comparative sample are shown in gray symbol. The Figure is reproduced from Paudel et al. (2018). In right, we show a relation between star-formation rate and B-band absolute magnitude, where the comparative sample galaxies taken from Lee et al.

derived value of the star-formation rate is significantly lower than the FUV value. This is because, area coverage of FUV is substantially more extensive compared to the SDSS 3" central region. This implies that, only a fraction of starformation happens in the center of the galaxy.

The emission line metallicity of SDSSJ134326.99+431118.7 is 7.85 dex, which is a typical for metal-poor the vigorously star-forming galaxies, called Blue-Compact Dwarf galaxies (BCDs) [15]. As their name indicates, they are significantly blue and gas-rich compact galaxies, mainly found in a very low-density environment. Comparing morphological and physical properties of SDSSJ134326.99+431118.7 with that of BCDs, it is clear that SDSSJ134326.99+431118.7 is a typical BCDs type galaxy formed by the merger in an isolated environment.

\section{Conclusion}

The emission-line spectrum of a system of interacting dwarf galaxy SDSS
$\mathrm{J} 134326.99+431118.7$ is studied. We noticed 21 spectral lines in the spectrum. Among them, only eight most robust emission-lines are analyzed in this work by using Gaussian fits. The following results are concluded from the observations and calculations.

a) We fitted the observed emission lines on the optical spectrum of SDSS J134326.99+431118.7 and found that there is an excellent agreement of all eight characteristic lines with Gaussian fits with more than $98 \%$ coefficient of regression.

b) We derived star-formation rate and emission line metallicity of SDSS J134326.99+431118.7, and found that the galaxy does not follow the scaling relation of $\mathrm{Mb}$ versus SFR and $\mathrm{Mb}$ versus $12+$ $\log (\mathrm{O} / \mathrm{H})$.

c) The morphological and physical properties of SDSSJ134326.99+431118.7 are very similar to those of a typical BCD galaxy. We conclude that we have presented another evidence of forming a BCD-type galaxy through a merger.

\section{Acknowledgements}

Daya Nidhi Chhatkuli acknowledge the University Grants Commission of Nepal, for financial support 
by providing $\mathrm{PhD}$ Fellowship and Research Support Grant (Award No.: PhD-75/76-S \& T-13) to carry out this research.

This study is based on the archival images and spectra from the Sloan Digital Sky Survey (http://www:sdss.org/collaboration/credits.html).

\section{References}

[1] F. Schweizer, Colliding and merging galaxies. Science 231 (1986) 227-234. https://science.sciencemag.org/content/231/4735/227

[2] S. Pearson, G. Besla, M. E. Putman, K. A. Lutz, X. Fernández, S. Stierwalt, ... \& E. C. Sung, Local Volume TiNy Titans: gaseous dwarf-dwarf interactions in the Local Universe, Monthly Notices of the Royal Astronomical Society 459 (2016) 1827-1846. https://doi.org/10.1093/mnras/stw757

[3] J. E. Barnes, \& L. Hernquist, Transformations of galaxies, II. Gasdynamics in merging disk galaxies. The Astrophysical Journal, 471(1996)(1),(115-142). https://iopscience.iop.org/article/10.1086/177957/meta

[4] K. Bekki, Formation of Blue Compact Dwarf Galaxies From Merging and Interacting gas-rich Dwarfs, Monthly Notices of the Royal Astronomical Society: Letters 388(2008) (1) L10-L14. https://academic.oup.com/mnrasl/article/388/1/L10/ 977927

[5] S. Paudel, C. Sengupta, \& S. J. Yoon, KUG 0200096: Dwarf Antennae Hosting a Tidal Dwarf Galaxy, The Astronomical Journal 156(2018) (4)(17). https://doi.org/10.3847/1538-3881/aadb8d

[6] G. De Lucia, V. Springel, S. D. White, D. Croton, \& G. Kauffmann, The Formation History of Elliptical Galaxies, Monthly Notices of the Royal Astronomical Society 366(2006) (2) 499-509. https://doi.org/10.1111/j.1365-2966.2005.09879.x

[7] S. Stierwalt, G. Besla, D. Patton, K. Johnson, N. Kallivayalil, M. Putman, ... \& G. Ross, TiNy Titans:
The Role of dwarf-dwarf Interactions in low-mass Galaxy Evolution. The Astrophysical Journal 805 (2015) 1-16 http://dx.doi.org/10.1088/0004-637X/805/1/2

[8] C. J. Conselice, C. Yang, \& A. F. Bluck, The Structures of Distant Galaxies-III. The Merger History of Over 20000 Massive Galaxies at $\mathrm{z}<1.2$, Monthly Notices of the Royal Astronomical Society 394 (2009) 1956-1972. https://doi.org/10.1111/j.1365-2966.2009.14396.x

[9] S. Stierwalt, G. Besla, D. R. Patton, K. E. Johnson, N. Kallivayalil, M. E. Putman,... \& G. Ross, The Role of Dwarf-Dwarf Interactions in the Evolution of Low Mass Galaxies, In American Astronomical Society Meeting Abstracts\# 225(2015). http://csma31.csm.jmu.edu/physics/courses/391/Spe akers/2015Spring/abstract_stierwalt_sabrina.pdf

[10] F. Lelli, F. Fraternali, \& M. Verheijen, Evolution of Dwarf Galaxies: a Dynamical Perspective, Astronomy \& Astrophysics 563 (2014) 1-27. http://dx.doi.org/10.1051/0004-6361/201322658

[11] S. Paudel, R. Smith, S. J. Yoon, P. Calderón-astillo, \& P. A. Duc, A Catalog of Merging Dwarf Galaxies in the Local Universe. The Astrophysical Journal Supplement Series 237 (2018) 1-20. https://doi.org/10.3847/1538-4365/aad555

[12] G. L. Squires \& Squires, G. L. Practical physics. Cambridge University press (2001).

[13] Jr. Kennicutt, C. Robert, Star Formation in Galaxies Along the Hubble Sequence, Annual Review of Astronomy and Astrophysics 36 (1998) 189-231.

[14] R. A. Marino et al., "The O3N2 and N2 abundance indicators revisited: improved calibrations based on CALIFA and Te-based literature data," Astronomy and Astrophysics vol. 559 (Nov. 2013) 114. https://doi.org/10.1051/0004-6361/201321956.

[15] Lee, J.C., Kennicutt, R.C., Jr., Funes, S.J.J.G., Sakai, S., Akiyama, S.. Dwarf Galaxy Starburst Statistics in the Local Volume, The Astrophysical Journal $692 \quad$ (2009) 1305-1320. https://doi.org/10.1088/0004-637X/692/2/1305 